\title{
Gate-Controlled Electron Quantum Interference Logic Devices
}

\author{
Josef Weinbub*1, Mauro Ballicchia², and Mihail Nedjalkov² \\ ${ }^{1}$ Christian Doppler Laboratory for High Performance TCAD, Institute for Microelectronics, TU Wien, Austria \\ ${ }^{2}$ Institute for Microelectronics, TU Wien, Austria
}

Recent advances in electron quantum optics $[1-10]$ show the breathtaking progress in utilizing the electron's wave nature. Inspired by these advances, we propose a new type of electron quantum interference logic device (eQILD), where an electron wave is coherently injected into a two-dimensional (2D) wave guide and controlled via two gates. Interference effects lead to different current levels in output channels and are utilized for classical logic gates. The operating principle is shown by means of dynamic quantum Wigner and classical simulations considering coherent/ballistic transport. Contrary to other advanced information processing approaches no magnetism nor bosonic systems are required [11]. The eQILD is inherently compatible with conventional integrated circuits and thus provides an attractive alternative towards advanced low-power information processing devices with the performance only limited by the single-electron source frequency being in the $\mathrm{GHz}$ regime [2]

Quantum interference devices have a long history, ranging from superconducting applications [12-14] to early investigations into logic [15]. In contrast, the eQILD uses a different operational principle (Figure 1). For the $2 \mathrm{D}$ wave guide we consider a $22 \times 40 \mathrm{~nm}^{2}$ molybdenum disulfide $\left(\mathrm{MoS}_{2}\right)$ single-layer; a 2D semiconductor of the family of transition metal dichalcogenides (TMDs). The initial choice of $\mathrm{MoS}_{2}$ is based on excellent transport, fabrication, and insulator compatibility properties and its wide-spread research focus $[16,17]$. However, the choice of wave guide material is not limited to $\mathrm{MoS}_{2}$, other types of 2D semiconductors [16] or 2D electron gases (2DEGs; e.g. (Al)GaAs heterostructures) are in principle compatible with the eQILD design. The wave guide is conceptually insulated by a cap and a substrate layer on the top and bottom, respectively, and offers reflective boundary conditions along $x=0 \mathrm{~nm}$ and $x=22 \mathrm{~nm}$ (Figure 2 and 3 ). The gates are embedded in the cap layer: Based on the charge and shape of the gates and the distance between them, the transport domain, the output channel walls (potential energy of $0.1 \mathrm{eV}$ and dimensions $2 \times 10 \mathrm{~nm}^{2}$ centered at $x=7 \mathrm{~nm}$ and $15 \mathrm{~nm}$, respectively, and ranging from $y=30-40 \mathrm{~nm}$ ), and the boundaries, we calculate the corresponding potential energy distribution in the

*josef.weinbub@tuwien.ac.at

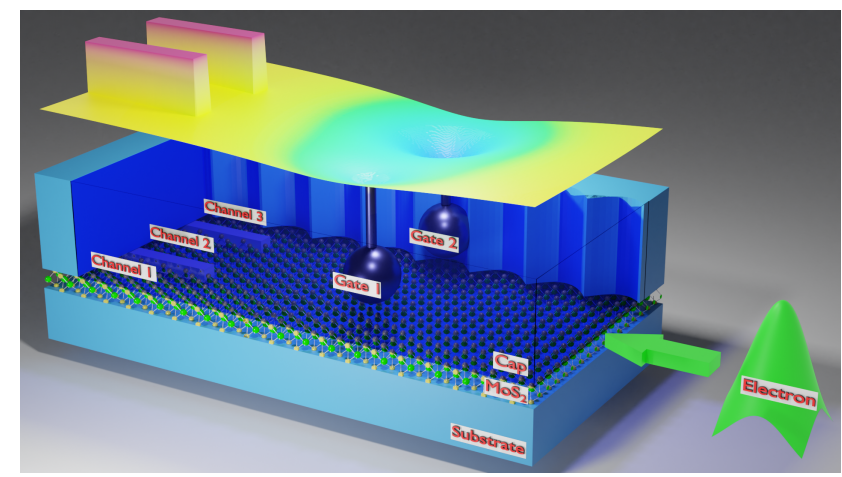

Figure 1: Schematic representation of the eQILD. An exemplary potential energy distribution of the wave guide derived from biased gates and the device structure is shown on top. The blue cap layer is partially made transparent to show the inside of the device.

channel as is discussed below (see an exemplary potential energy distribution overlaid in Figure 1). Further considering the physical characteristics of the injected electrons, we use the calculated potential energy distribution to simulate the electron transport (quantum and classical) in the 2D wave guide by stochastic evolution simulations [18] (see Supplementary Information A) for various gate configurations and calculate the current levels in the output channels.

The simulation setup conceptually resembles a double-slit experiment, where single electrons are shot consecutively towards a screen, until a stationary (steady-state) interference pattern is obtained [19,20]. The probabilistic interpretation is of equivalent independent (uncorrelated) experiments aiming at accumulating sufficient statistics. Electrons with energies of $71 \mathrm{meV}$ enter on one side of the device and have a dwelling time in the femtosecond regime, underlining the sole limitation of performance on the electron source frequency [2].

Single-layer $\mathrm{MoS}_{2}$ is a direct-bandgap (1.8 eV) semiconductor, which makes it well-suited for low-power applications [21]. First principles band structure calculations showed that for n-type single-layer $\mathrm{MoS}_{2}$ the $\mathrm{K}$, $\mathrm{K}$ 'valleys, which are well-separated in energy from the satellite valleys, are most relevant for low-field transport. A parabolic band with electron mass $m=0.48 m_{0}$ (see (1) in Supplementary Information A), with $m_{0}$ being the mass of the free electron, gives a perfect description of the conduction band valley around the $K$ point. 


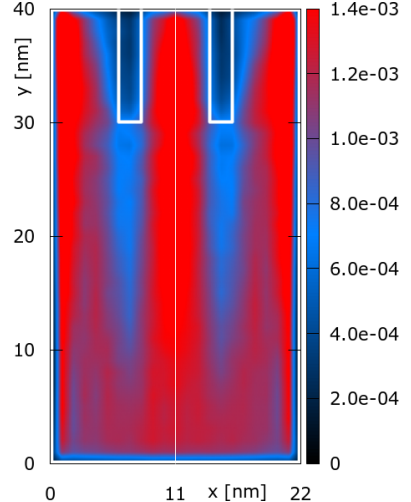

(a)

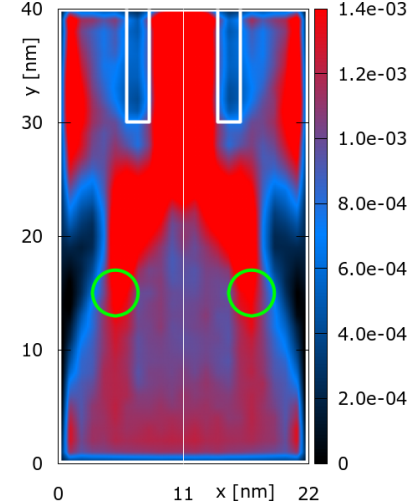

(b)
Figure 2: Distribution of quantum electron density [a.u.] for two symmetric gate configurations: $\phi_{G_{1}}=$ $\phi_{G_{2}}=0 \mathrm{~V}$ (a) and $\phi_{G_{1}}=\phi_{G_{2}}=0.21 \mathrm{~V}$ (b). The output channel walls are shown in white. Green circles indicate location of gates.
The metal gates are each modeled as solid spheres with a radius of $2 \mathrm{~nm}$ positioned $3 \mathrm{~nm}$ (relative to the sphere center) above the wave guide, resulting in $1 \mathrm{~nm}$ cap oxide material (calcium fluoride $-\mathrm{CaF}_{2}[16]$ ) between the surface of the gates and the $\mathrm{MoS}_{2}$. In the following discussions of the calculated $2 \mathrm{D}$ quantum electron densities in the wave guide we consider various gate bias configurations in terms of the electric potential $\phi$ (potential energy $V=-q \phi$ with $q$ the electron charge): A negative or positive gate bias represents a repulsive barrier or an attractive well for the electrons, respectively.

Figure 2a considers no applied bias to the gates, Gate $1\left(G_{1}\right)$ and Gate $2\left(G_{2}\right)$. Electrons are injected in $y$-direction at the bottom (see green wave-packet in Fig. 1). The two wall potentials impose strong nonlocal effects upon the electrons, shown in particular by the reduced density in y-direction below the walls. The electron density is thus equally split into the three output channels, following the symmetry of the walls. Figure $2 \mathrm{~b}$ shows the electron density for a symmetric gate bias configuration, i.e., $\phi_{G_{1}}=\phi_{G_{2}}=0.21 \mathrm{~V}$. The resulting peak of the superimposed electric potential of the two gates, as determined by the gate and device geometry, is $0.17 \mathrm{~V}$ on the $\mathrm{MoS}_{2}$ surface, i.e., both gates act as attractive wells. As a result, a focusing effect materializes: The majority of the electron density is guided towards output channel 2 (the central channel).

Figure 3a shows the electron density for an asymmetric gate bias configuration, i.e., $\phi_{G_{1}}=0 \mathrm{~V}$ and $\phi_{G_{2}}=0.21 \mathrm{~V}$, corresponding to a resulting peak superimposed electric potential on the $\mathrm{MoS}_{2}$ surface of $0.14 \mathrm{~V}$, i.e., $\phi_{G_{2}}$ acts as an attractive well in front of electron density is thus guided towards channel 3, less so towards channel 2 , and considerably less to channel 1 (left channel). Figure 3b shows the electron density for another representative asymmetric gate configuration where the gates are biased in an opposing manner, output channel 3 (right channel): The majority of the

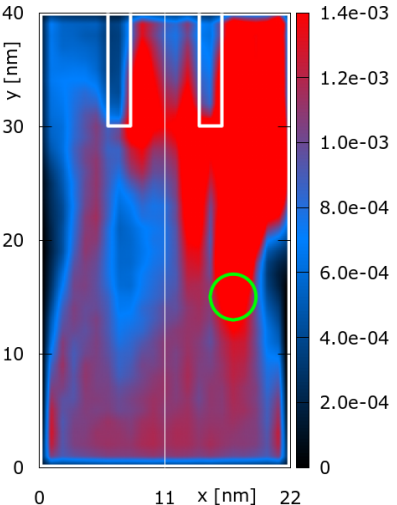

(a)

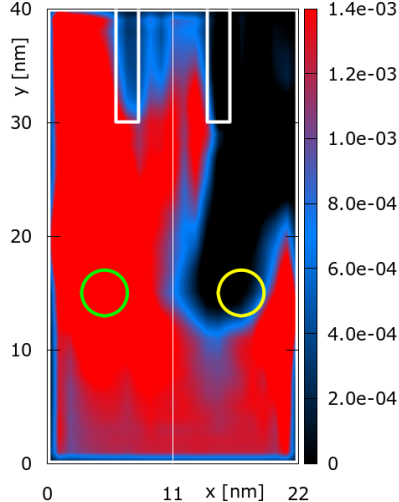

(b)
Figure 3: Distribution of quantum electron density [a.u.] for two asymmetric gate configurations: $\phi_{G_{1}}=$ $0 \mathrm{~V}$ and $\phi_{G_{2}}=0.21 \mathrm{~V}$ (a) and $\phi_{G_{1}}=0.21 \mathrm{~V}$ and $\phi_{G_{2}}=-0.21 \mathrm{~V}(\mathrm{~b})$. The yellow circle indicates the location of the negatively charged gate.

i.e., $\phi_{G_{1}}=0.21 \mathrm{~V}$ and $\phi_{G_{2}}=-0.21 \mathrm{~V}$. This configuration corresponds to a resulting peak superimposed electric potential on the $\mathrm{MoS}_{2}$ surface of $0.11 \mathrm{~V}$ in front of channel 1 , and a repulsive barrier of $-0.11 \mathrm{~V}$ in front of channel 3. In this case the electron density guided towards channel 3 is almost completely blocked and the majority of the electron moves towards channel 1; a considerably smaller part of the electron density enters channel 2. The effects of penetration and tunneling into the output channel walls seen in both cases of Figure 3 is a clear indication of the quantum character of the electron evolution.

Figure 4 shows the calculated quantum currents for a representative selection of $\phi_{G_{1}}$ biases relative to fixed $\phi_{G_{2}}$ biases in the three output channels. The current at the output of each channel has been calculated by integrating the current density along the corresponding output cross-section. In turn, the current density is calculated from the first-order moment of the electron distribution function. Figure 4a considers the case of $\phi_{G_{2}}=0 \mathrm{~V} ; G_{2}$ is thus not active (see a similar scenario in Figure 3a albeit for $\left.G_{1}\right)$. For negative $\phi_{G_{1}}$ biases, $\phi_{G_{1}}$ repulses the majority of the current to channel 3, with a similar inverted behavior for positive biases with respect to channel 1 . In case of $\phi_{G_{1}}=0 \mathrm{~V}$, the electron is equally distributed between all three output channels as expected from device symmetry considerations, which corresponds to the scenario depicted in Figure 2a. The negative and positive $\phi_{G_{1}}$ bias ranges seem to offer a mirrored behavior, however, both ranges offer entirely different physical scenarios of repulsion and attraction, leading to different interactions between the electrons and the potential energy landscape and thus to different current levels. Therefore, a fully-mirrored behavior is not to be expected. Figure $4 \mathrm{~b}$ considers the case of $\phi_{G_{2}}=0.105 \mathrm{~V}$. The negative bias range offers similar general behavior as for $\phi_{G_{2}}=0 \mathrm{~V}$ (see Figure 4a), however, for the symmetric case $\phi_{G_{1}}=\phi_{G_{2}}=0.105 \mathrm{~V}$ a focusing effect with respect to channel 2 manifests (similar to Figure $2 b$ ). 
A further increased $\phi_{G_{1}}$ bias results in a deeper well potential at $G_{1}$ over $G_{2}$, thus the majority of the current manifests in channel 1. Figure 4c considers the case of $\phi_{G_{2}}=0.21 \mathrm{~V} ; G_{2}$ acts as an even stronger attractive well. Yet another intriguing example of non-locality is seen here for negative biases: Although $\phi_{G_{2}}$ has doubled (compare with Figure $4 \mathrm{~b}$ ), the current in channel 1 \& 2 stays roughly the same but in channel 3 (located behind $G_{2}$ ) is reduced further, demonstrating the wave nature of the evolution.

Table 1 provides an extended overview of the gate bias to channel current relations and together with Table 2 shows various exemplary interpretations as logic gates: Particularly important are universal NAND and NOR gates as both are functionally complete and as such can be used to implement all possible Boolean functions [22]. In classical logic, a NAND gate is defined by the truth table $00 \rightarrow 1,01 \rightarrow 1,10 \rightarrow 1$, $11 \rightarrow 0$ and the NOR gate by $00 \rightarrow 1,01 \rightarrow 0$, $10 \rightarrow 0,11 \rightarrow 0$, mapping two input states to one output state for the binary-based four configurations. In a similar fashion, additional logic gates can be identified, e.g., AND and NOT gates.

Comparative classical simulations (see Supplementary Information A) verified that the current is significantly (15-51\%) larger in the quantum case. The increased current is due to the wave nature of the electrons, in particular constructive interference. For example, when both gates are driven by $0.105 \mathrm{~V}, I_{C_{2}}=$ $46 \mu \mathrm{A}$ but the classical current is $28 \mu \mathrm{A}(\sim 39 \%$ reduction). An even stronger difference is observed for $0.21 \mathrm{~V}$, where $I_{C_{2}}=41 \mu \mathrm{A}$ against a classical current of $20 \mu \mathrm{A}(\sim 51 \%$ reduction). A similar behavior is also present for repulsive symmetric potentials: For $-0.105 \mathrm{~V}, I_{C_{2}}=31 \mu \mathrm{A}$ is reduced to $21 \mu \mathrm{A}$ in the classical case ( $\sim 32 \%$ reduction). Furthermore, in the asymmetric case of $\phi_{G_{2}}=0.105 \mathrm{~V}$ and $\phi_{G_{2}}=$ $-0.105 \mathrm{~V}, I_{C_{1}}=39 \mu \mathrm{A}$ while the classical current is $33 \mu \mathrm{A}(\sim 15 \%$ reduction). These significant differences between the quantum and the classical currents clearly show, that indeed quantum effects are essential for eQILDs to allow for more distinct and thus practically usable current levels.

In conclusion, the introduced eQILD provides an alternative path towards advanced non-magnetic, lowpower, and high performance classically operating logic devices. The electronic nature of eQILDs provides an attractive alternative path towards co-integration with conventional electronics. The device structure is amenable for fabrication due to simplistic geometries and established materials. Furthermore, various adaptations are perceivable, for instance, additional gates, channels, or bias configurations would allow to introduce even more current states. Alternatively, the output channels can be arranged in a non-symmetric manner or can be merged outside of the device to allow for further superpositioned states. Robustness is tuneable by increasing the current ranges. The eQILD design is in principle adaptable to other transport materials (e.g. other TMDs, 2DEGs), offering additional options for practical realizations. In addition to classical bi- nary logic, the manifestation of several current levels at the individual output channels provides a path towards multi-valued logic, which, although having inherent higher robustness challenges [22], promises higher integration densities [23]. A particular attractive perspective is provided considering the recent advances towards two-electron sources [5]: The eQILD operation could be extended towards applications in entangletronics [19,24,25], enabling the use of entanglement for non-local classical logic.

\begin{tabular}{|c|c|c|c|c|c|}
\hline$\phi_{G_{1}}$ & $\phi_{G_{2}}$ & $I_{C_{1}}$ & $I_{C_{2}}$ & $I_{C_{3}}$ & GATE \\
\hline-0.21 & -0.21 & 9 & 10 & 9 & NOR: $11 \rightarrow 0$ \\
-0.21 & -0.105 & 10 & 14 & 28 & \\
-0.21 & 0 & 13 & 15 & 36 & NOR: $10 \rightarrow 0$ \\
-0.21 & 0.105 & 16 & 13 & 37 & \\
-0.21 & 0.21 & 15 & 14 & 32 & \\
-0.105 & -0.21 & 28 & 14 & 10 & \\
-0.105 & -0.105 & 19 & 31 & 19 & NAND: $00 \rightarrow 1$ \\
-0.105 & 0 & 17 & 11 & 47 & \\
-0.105 & 0.105 & 18 & 13 & 39 & NAND: $01 \rightarrow 1$ \\
-0.105 & 0.21 & 16 & 16 & 24 & \\
0 & -0.21 & 36 & 15 & 13 & NOR: $01 \rightarrow 0$ \\
0 & -0.105 & 47 & 11 & 17 & \\
0 & 0 & 26 & 27 & 26 & NOR: $00 \rightarrow 1$ \\
0 & 0.105 & 17 & 13 & 47 & \\
0 & 0.21 & 14 & 20 & 34 & \\
0.105 & -0.21 & 37 & 13 & 16 & \\
0.105 & -0.105 & 39 & 13 & 18 & NAND: $10 \rightarrow 1$ \\
0.105 & 0 & 47 & 13 & 17 & \multirow{2}{*}{ NAND: $11 \rightarrow 0$} \\
0.105 & 0.105 & 15 & 46 & 15 & NAN 1 \\
0.105 & 0.21 & 10 & 20 & 40 & \\
0.21 & -0.21 & 32 & 14 & 15 & \\
0.21 & -0.105 & 24 & 16 & 16 & \\
0.21 & 0 & 34 & 20 & 14 & \\
0.21 & 0.105 & 40 & 20 & 10 & \\
0.21 & 0.21 & 15 & 41 & 15 & \\
\hline & & & &
\end{tabular}

Table 1: Output channel quantum currents $I_{C_{1,2,3}}(\mu \mathrm{A})$ for an extended set of gate biases $\phi_{G_{1,2}}(\mathrm{~V})$ relative to Figure 4. The GATE column shows individual rows of the truth tables of realizable NAND and NOR gates according to the rules shown in Table 2; framed current values highlight the utilized channel to realize the logic.

\begin{tabular}{|c|c|c|c|c|c|}
\hline \multicolumn{2}{|c|}{ IN } & C & \multicolumn{2}{c|}{ OUT } & GATE \\
0 & 1 & & 0 & 1 & \\
\hline 0 & -0.21 & 2 & $\leq 20$ & $>20$ & NOR \\
-0.105 & 0.105 & 3 & $\leq 16$ & $>16$ & NAND \\
\hline
\end{tabular}

Table 2: Exemplary logic gate definitions based on $\phi_{G}$ and $I_{C}$ values of Table 1: IN associates specific $\phi_{G_{1,2}}$ (V) biases with input states 0 and 1 ; $\mathrm{C}$ is the output channel number; OUT associates specific $I_{C_{1,2,3}}$ thresholds $(\mu \mathrm{A})$ with output states 0 and 1 ; and GATE shows the realizable logic gate. 


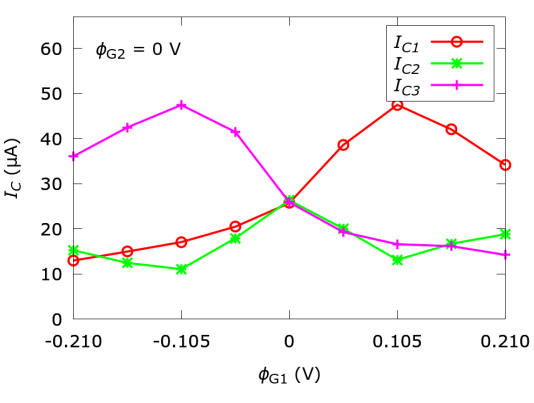

(a)

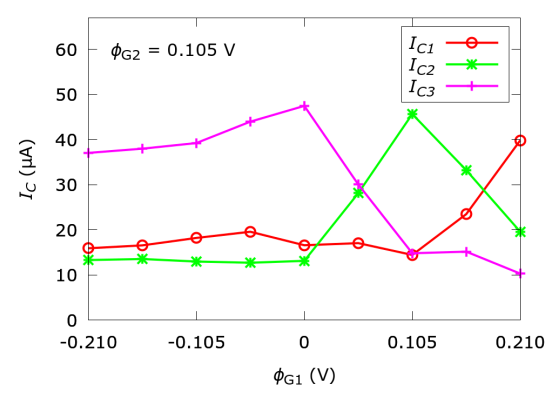

(b)

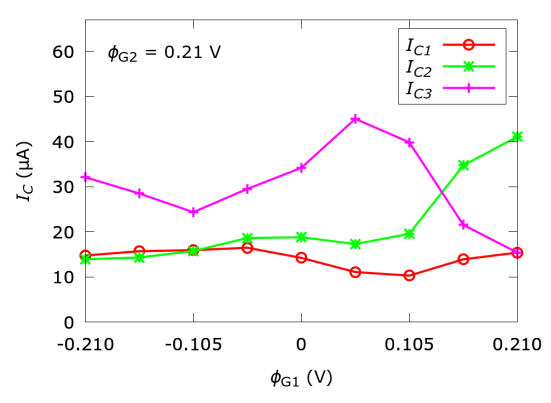

(c)

Figure 4: Output channel quantum currents $I_{C_{1,2,3}}$ for a set of gate biases $\phi_{G_{1,2}}$.

\section{References}

[1] Bäuerle, C. et al. Coherent Control of Single Electrons: A Review of Current Progress. Reports on Progress in Physics 81, 056503 (2018). doi:10.1088/1361-6633/aaa98a.

[2] Yamahata, G. et al. Picosecond Coherent Electron Motion in a Silicon Single-Electron Source. Nature Nanotechnology 14, 1019-1023 (2019). doi:10.1038/s41565-019-0563-2.

[3] Bisognin, R. et al. Quantum Tomography of Electrical Currents. Nature Communications 10, 3379 (2019). doi:10.1038/s41467-019-11369-5.

[4] Fletcher, J. D. et al. Continuous-Variable Tomography of Solitary Electrons. Nature Communications 10, 5298 (2019). doi:10.1038/s41467-019-13222-1.

[5] Moskalets, M., Kotilahti, J., Burset, P. \& Flindt, C. Composite Two-Particle Sources. The European Physical Journal Special Topics 229, 647-662 (2020). doi:10.1140/epjst/e2019-900121-x.

[6] Rodriguez, R. H. et al. Relaxation and Revival of Quasiparticles Injected in an Interacting Quantum Hall Liquid. $\mathrm{Na}$ ture Communications 11, 2426 (2020). doi:10.1038/s41467020-16331-4.

[7] Clark, L. A., Kataoka, M. \& Emary, C. Mitigating Decoherence in Hot Electron Interferometry. New Journal of Physics 22, 103031 (2020). doi:10.1088/1367-2630/abb9e5.

[8] Bellentani, L., Forghieri, G., Bordone, P. \& Bertoni, A. Two-Electron Selective Coupling in an Edge-State Based Conditional Phase Shifter. Physical Review B 102, 035417 (2020). doi:10.1103/PhysRevB.102.035417.

[9] Lepage, H. V., Lasek, A. A., Arvidsson-Shukur, D R. M. \& Barnes, C. H. W. Entanglement Generation via Power-of-Swap Operations Between Dynamic ElectronSpin Qubits. Physical Review A 101, 022329 (2020). doi:10.1103/PhysRevA.101.022329.

[10] Ito, R. et al. Coherent Beam Splitting of Flying Electrons Driven by a Surface Acoustic Wave. Physical Review Letters 126, 070501 (2021). doi:10.1103/PhysRevLett.126.070501.

[11] International Roadmap for Devices and Systems (IRDS): Beyond CMOS (2020). URL https://irds.ieee.org/ editions $/ 2020$.

[12] Jaklevic, R. C., Lambe, J., Silver, A. H. \& Mercereau, J. E. Quantum Interference Effects in Josephson Tunneling. Physical Review Letters 12, 159-160 (1964). doi:10.1103/PhysRevLett.12.159.

[13] de Graaf, S. E. et al. Charge Quantum Interference Device. Nature Physics 14, 590-594 (2018). doi:10.1038/s41567018-0097-9.

[14] Braginski, A. I. Superconductor Electronics: Status and Outlook. Journal of Superconductivity and Novel Magnetism 32, 23-44 (2019). doi:10.1007/s10948-018-4884-4.

[15] Cahay, M. \& Bandyopadhyay, S. Semiconductor Quantum Devices. vol. 89 of Advances in Electronics and Electron Physics, 93-253 (Academic Press, 1994). doi:10.1016/S0065-2539(08)60074-8.

[16] Illarionov, Y. Y. et al. Insulators for 2D Nanoelectronics: The Gap to Bridge. Nature Communications 11, 3385 (2020). doi:10.1038/s41467-020-16640-8.
[17] Ahn, C. et al. Growth of Monolayer and Multilayer MoS2 Films by Selection of Growth Mode: Two Pathways via Chemisorption and Physisorption of an Inorganic Molecular Precursor. ACS Applied Materials \&S Interfaces 13, 68056812 (2021). doi:10.1021/acsami.0c19591.

[18] Weinbub, J. \& Ferry, D. Recent Advances in Wigner Function Approaches. Applied Physics Reviews 5, 041104 (2018). doi:10.1063/1.5046663.

[19] Weinbub, J., Ballicchia, M. \& Nedjalkov, M. Electron Interference in a Double-Dopant Potential Structure. Physica Status Solidi RRL 12, 1800111 (2018). doi:10.1002/pssr.201800111.

[20] Ballicchia, M., Weinbub, J. \& Nedjalkov, M. Electron Evolution Around a Repulsive Dopant in a Quantum Wire: Coherence Effects. Nanoscale 10, $23037-23049$ (2018). doi:10.1039/C8NR06933F.

[21] Kaasbjerg, K., Thygesen, K. S. \& Jacobsen, K. W. Phonon-Limited Mobility in $n$-Type Single-Layer $\mathrm{MoS}_{2}$ from First Principles. Physical Review B 85, 115317 (2012). doi:10.1103/PhysRevB.85.115317.

[22] Mano, M. M., Kime, C. R. \& Martin, T. Logic and Computer Design Fundamentals, 5th Edition (Pearson, 2016).

[23] Lee, L. et al. $\mathrm{ZnO}$ Composite Nanolayer with Mobility Edge Quantization for Multi-Value Logic Transistors. Nature Communications 10, 1998 (2019). doi:10.1038/s41467019-09998-x.

[24] Ellinghaus, P., Weinbub, J., Nedjalkov, M. \& Selberherr, S. Analysis of Lense-Governed Wigner Signed Particle Quantum Dynamics. Physica Status Solidi RRL 11, 1700102 (2017). doi:10.1002/pssr.201700102.

[25] Benam, M., Ballicchia, M., Weinbub, J., Selberherr, S. \& Nedjalkov, M. A Computational Approach for Investigating Coulomb Interaction using Wigner-Poisson Coupling. Journal of Computational Electronics (2021). doi:10.1007/s10825-020-01643-x.

[26] Nedjalkov, M. et al. Wigner Equation for General Electromagnetic Fields: The Weyl-Stratonovich Transform. Physical Review B 99, 014423 (2019). doi:10.1103/PhysRevB.99.014423.

[27] Querlioz, D., Dollfus, P. \& Mouis, M. The Wigner Monte Carlo Method for Nanoelectronic Devices (Wiley-ISTE, Hoboken-London, 2010). doi:10.1002/9781118618479.

[28] Nedjalkov, M., Querlioz, D., Dollfus, P. \& Kosina, H. Wigner Function Approach, 289-358 (Springer, New York, 2011). doi:10.1007/978-1-4419-8840-9_5.

[29] Ellinghaus, P., Weinbub, J., Nedjalkov, M., Selberherr, S. \& Dimov, I. Distributed-Memory Parallelization of the Wigner Monte Carlo Method Using Spatial Domain Decomposition. Journal of Computational Electronics 14, 151162 (2015). doi:10.1007/s10825-014-0635-3.

[30] Ryu, S., Kataoka, M. \& Sim, H.-S. Ultrafast Emission and Detection of a Single-Electron Gaussian Wave Packet: A Theoretical Study. Physical Review Letters 117, 146802 (2016). doi:10.1103/PhysRevLett.117.146802. 


\section{A Supplementary Information}

\section{Modelling Theory}

The numerically solved Wigner transport equation is

$$
\begin{aligned}
& {\left[\frac{\partial}{\partial t}+\frac{\boldsymbol{p}}{m} \cdot \frac{\partial}{\partial \boldsymbol{r}}\right] f_{W}(\boldsymbol{p}, \boldsymbol{r}, t)=} \\
& \int d \boldsymbol{p}^{\prime} V_{W}\left(\boldsymbol{p}-\boldsymbol{p}^{\prime}, \boldsymbol{r}\right) f_{W}\left(\boldsymbol{p}^{\prime}, \boldsymbol{r}, t\right)
\end{aligned}
$$

and corresponds to spatially dependent, electric fields slowly varying in time, so that magnetic effects are not involved [26]. Here, $t$ is the time, $m$ the electron mass, $\boldsymbol{p}$ the momentum, and $\boldsymbol{r}$ the spatial position; $(\boldsymbol{p}, \boldsymbol{r})$ spans the phase space. Furthermore, $V_{W}$ is the Wigner potential operator obtained from the electric potential $\phi(\boldsymbol{r})$ by the Weyl transform and $f_{W}$ is the Wigner function, providing physical averages by the same expressions inherent for the classical distribution function. The equation fully accounts for coherent, time-dependent quantum effects, such as tunnelling, interference, and non-locality. Despite that (1) can be augmented by terms accounting for decoherence effects of scattering [27] here we focus on the coherent behavior, governed by the complicated interplay of the physical characteristics of the electron state in terms of velocity, energy and dispersion, electric potentials and geometry.

We apply the signed-particle model to the Wigner transport equation [28]. This model extends the conventional, classical particle model applied in the Boltzmann theory by assigning quantum information to the particles. Quantum particles evolve in a classical way, however, they carry a plus or minus sign, are generated by the Wigner potential $V_{W}$, and annihilate each other if they occupy the same point in phase space. Ultimately, we solve the signed-particle Wigner transport equation by a stochastic approach, provided by our ensemble Monte Carlo solver VIEnNA WD ${ }^{1}$ [29].

The classical limit of (1) is obtained in the case of slowly varying potentials, when the Wigner potential reduces to the classical force giving rise to the ballistic Boltzmann equation [28]. Accordingly, the signedparticle model reduces to the Boltzmann model of classically evolving particles.

An electron is modelled as a minimum uncertainty wave packet, which is described by an admissible Wigner pure state [27]

$$
\begin{aligned}
f_{W}(\boldsymbol{r}, \boldsymbol{p})= & N \exp \left\{-\left|\boldsymbol{r}-\boldsymbol{r}_{0}\right|^{2} /\left(2 \sigma^{2}\right)\right\} \\
& \exp \left\{-\left|\boldsymbol{p}-\boldsymbol{p}_{0}\right|^{2} 2 \sigma^{2} / \hbar^{2}\right\},
\end{aligned}
$$

where $N$ is a normalization constant. The state (2) is characterized by a Gaussian distribution [30] with a standard spatial deviation $\sigma=16 \mathrm{~nm}$. This corresponds to a momentum deviation smaller than $0.1 \mathrm{~nm}^{-1}$ around $p_{0}=0.94 \mathrm{~nm}^{-1}$ and also to an uncertainty of about $5 \mathrm{meV}$ with respect to the kinetic energy of the injected electron.

\footnotetext{
$1_{\text {www .iue.tuwien.ac.at/software/viennawd/ }}$
}

\section{Acknowledgements}

The financial support by the Austrian Science Fund (FWF): P29406 and P33609, the Austrian Federal Ministry for Digital and Economic Affairs and the National Foundation for Research, Technology and Development is gratefully acknowledged. The computational results presented here have been achieved using the Vienna Scientific Cluster (VSC). 